\title{
Thermo-hydro-mechanical coupling in clay barriers
}

\author{
F. Collin*, X.L. Li, J.P. Radu, R. Charlier \\ Department GeomaC, University of Liège, Sart Tilman B52/3, Chemin des Chevreuils, 1, B-4000, Liege, Belgium
}

Received 10 March 2000; accepted 23 November 2001

\begin{abstract}
A thermo-hydro-mechanical model is presented to tackle the complex coupling problems encountered in clay barriers. A detailed formulation coupling heat, moisture (liquid water and water vapour) and air transfer in a deformable unsaturated soil is given. The formulation of Alonso-Gens' mechanical model for unsaturated soil is also incorporated. Finally, a small-scale wetting-heating test on compacted bentonite is performed for validation; the numerical results are compared to the experimental measurements. (C) 2002 Elsevier Science B.V. All rights reserved.
\end{abstract}

Keywords: Unsaturated soil; Suction; Multiphase flow; Numerical modelling

\section{Introduction}

One of nuclear waste disposal concepts proposes to store waste in deep impervious clay geological layers. Vitrified waste is laid in canisters in the middle of gallery dug in the clay formations. An engineered barrier generally made of highly compacted clay blocks fills the remaining surface of the shaft. The aim is the creation of the most compact, stable and impervious barrier. Engineered clay is initially unsaturated and undergoes a very high suction (up to 100 $\mathrm{MPa}$ or more). But the material is progressively wetted by the water table of the host formation. Moreover, waste still produces a certain amount of heat and the confinement barrier is subjected to high temperature (over $70{ }^{\circ} \mathrm{C}$ and sometimes over $100{ }^{\circ} \mathrm{C}$ ).

\footnotetext{
${ }^{*}$ Corresponding author. Tel.: +32-4-3669142; fax: +32-43669326.

E-mail address: F.Collin@ulg.ac.be (F. Collin).
}

A good design of a clay barrier should take all these phenomena into account. For this purpose, constitutive laws have been developed. They are coupling water flow, heat flow and soil mechanic and they have been implemented in a finite element code, which allows analysing non-homogenous transient problems.

The mechanical behaviour of an unsaturated soil depends on stress level and on suction (Fredlund, 1993). The mechanical constitutive law developed here is based on a refined model, which has been proposed 10 years ago by Alonso et al. (1990). On the other hand, unsaturated flow is a non-linear problem. Moreover, high temperature induces the production of water vapour (which depends also on the suction level). Our flow model is mainly based on previous works of Thomas and He (1995) and Olivella et al. (1994). The water vapour flow formulation follows Philip's and de Vries' (1957).

The developed finite elements are isoparametric elements with the following degrees of freedom: soil 
skeleton displacements, temperature, liquid water pressure, and gas (dry air + vapour) pressure. The elements have a monolithical form, and all coupling terms in the Newton-Raphson's stiffness matrix are taken into account, allowing a good convergence rate for most treated problems.

Finally, a validation of the constitutive laws and of the finite element code is obtained thanks to a comparison with results of an experiment presented in the paper.

\section{Diffusion model}

In clay barriers, unsaturated conditions and thermal variations create coupling effects that influence the design of each component of the barriers. Moreover, high temperatures in unsaturated conditions induce production of water vapour. Each phase (liquid and gaseous) constitutes a mixture of two components, which are dry air and water vapour for the gas phase and liquid water and dissolved air for the liquid phase.

The compositional approach (Panday and Corapcioglu, 1989) is used here to write balance equations: we assume the conservation mass of each chemical species (water and air). Using this approach, the phase exchange term will cancel in balance equations.

The variables chosen for the description of the flow problem are liquid water pressure, gas pressure and temperature.

\subsection{Water species}

The mass conservation equation of water species is obtained by summing the balance equation of liquid water and water vapour.

Clay presents a very low permeability and very slow liquid water motions. The effect of water vapour transport in this type of soil may not be neglected because vapour flows will highly influence moisture transfer.

\subsubsection{Mass conservation for the water}

The equation includes the variation of water storage and the divergence of water flows in each phase. Water vapour is one of the gas phase's compounds. Therefore, vapour flows have two contributions: the advective flux of the gaseous phase and the nonadvective flux of water vapour related to vapour diffusion inside the gaseous phase.

$$
\begin{aligned}
& \underbrace{\frac{\partial \rho_{\mathrm{w}} n S_{\mathrm{r}, \mathrm{w}}}{\partial t}+\operatorname{div}\left(\rho_{\mathrm{w}} \underline{f}_{\mathrm{w}}\right)}_{\text {Liquid water }} \\
& +\underbrace{\frac{\partial \rho_{\mathrm{v}} n S_{\mathrm{r}, \mathrm{g}}}{\partial t}+\operatorname{div}\left(\underline{i}_{\mathrm{v}} \rho_{\mathrm{v}} \underline{f}_{\mathrm{g}}\right)}_{\text {Water vapour }}=0
\end{aligned}
$$

where $\rho_{\mathrm{w}}$ is liquid water density, $n$ is medium porosity, $S_{\mathrm{r}, \mathrm{w}}$ is water saturation degree in volume, $f_{\text {in }}$ is macroscopic velocity of the phase $\alpha, \underline{i}_{\mathrm{v}}$ is the nonadvective flux of water vapour, $\rho_{\mathrm{v}}$ is water vapour density, $S_{\mathrm{r}, \mathrm{g}}$ is gas saturation degree in volume and $t$ is the time.

\subsubsection{Motion of the liquid water}

The generalised Darcy's law for multiphase porous medium gives liquid water velocity:

$f_{-\mathrm{w}}=-\frac{k_{\text {int }} k_{\mathrm{r}, \mathrm{w}}}{\mu_{\mathrm{w}}}\left[\underline{\nabla} p_{\mathrm{w}}+g \rho_{\mathrm{w}} \underline{\nabla} y\right]$

where $p_{\mathrm{w}}$ is the liquid water pressure; $y$ is the vertical, upward directed co-ordinate; $g$ is the gravity acceleration; $\mu_{\mathrm{w}}$ is the dynamic viscosity of the liquid water; $k_{\text {int }}$ is the intrinsic permeability of the medium; and $k_{\mathrm{r}, \mathrm{w}}$ is the water relative permeability.

The water permeability varies with respect to the saturation degree in unsaturated conditions: pores filled by air do not more constitute flow pathways of water and permeability is consequently decreased.

\subsubsection{Couplings between the liquid water and other variables}

The liquid water properties (i.e. density and viscosity) depend on temperature. This induces a coupling between liquid water flow and thermal flow: some convective water flows can be created due to temperature distribution. Another coupling effect is related to permeability, which depends on saturation. In the model, the saturation is defined by the retention curve, as a function of suction (i.e. the difference between the gas and water pressure). The suction field will then influence the water flows. 


\subsubsection{Diffusion of water vapour}

The water vapour flow is assumed to follow a Fick's diffusion law in a tortuous medium. The vapour diffusion is linked to gradient of vapour density and the vapour flow follows the formulation proposed by the model of Philip and de Vries (1957):

$\underline{i}_{\mathrm{v}}=-D_{\mathrm{atm}} \tau_{\mathrm{v}} n S_{\mathrm{r}, \mathrm{g}} \underline{\nabla} \rho_{\mathrm{v}}$

where $D_{\text {atm }}$ is the molecular diffusion coefficient and $\tau_{\mathrm{v}}$ is the tortuosity.

Vapour is assumed to be in equilibrium with liquid water and vapour density $\rho_{\mathrm{v}}$ is given by the following thermodynamic relationship (Edlefsen and Anderson, 1943):

$\rho_{\mathrm{v}}=\rho_{0} h$

where $\rho_{0}$ is the saturated water vapour density and $h$ is the relative humidity.

The relative humidity takes into account adsorption phenomena and capillary effect in the soil. The Kelvin-Laplace's law defines this parameter:

$h=\exp \left(\frac{s}{\rho_{\mathrm{w}} R_{\mathrm{v}} T}\right)$

where $R_{\mathrm{v}}$ is the gas constant of water vapour, $s$ is the suction $\left(s=p_{\mathrm{g}}-p_{\mathrm{w}}\right)$ and $T$ is the temperature.

The vapour is considered as a perfect gas and vapour pressure is computed thanks to the perfect gas law:

$p_{\mathrm{v}}=\rho_{\mathrm{v}} R_{\mathrm{v}} T$

The gradient of the water vapour density can now be developed from Eq. (4) in order to compute the vapour flow:

$$
\begin{aligned}
\underline{\nabla} \rho_{\mathrm{v}}= & \frac{\rho_{0} g h}{R_{\mathrm{v}} T} \underline{\nabla} \frac{p_{\mathrm{w}}-p_{\mathrm{g}}}{\rho_{\mathrm{w}} g} \\
& +\left[h \frac{\partial \rho_{0}}{\partial T}-\frac{\rho_{0}\left(p_{\mathrm{w}}-p_{\mathrm{g}}\right) h}{\rho_{\mathrm{w}} R_{\mathrm{v}} T^{2}}\right] \underline{\nabla} T
\end{aligned}
$$

The water vapour density gradient can be separated into two contributions: an isothermal one related to a suction gradient and a thermal one due to a temperature gradient.

\subsubsection{Couplings between the water vapour and other variables}

As shown above, vapour properties and flows depend essentially on temperature and on gas pressure fields. This model can reproduce the vapour transport from points at high temperature (where the water vapour is produced) to points at lower temperature (where the water vapour condenses).

\subsection{Dry air species}

Dry air is a component of the gas phase, which is a mixture of dry air and water vapour. Dissolved air in the water is taken into account by the model and Henry's law allows to define the volume of air present in the liquid water. The dry air pressure is not a basic variable; this pressure will be computed from the gas and the vapour pressure assuming the validity of Dalton's law. The pressure of the gas mixture is equal to the sum of the partial pressures, which each gas would exert if it filled alone all the volume considered.

\subsubsection{Mass conservation for the dry air}

The equation of mass conservation includes the contributions of dry air and dissolved air in water. The dry air flows have two contributions: an advective flux related to gas phase motion and a non-advective flux corresponding to air diffusion in the gaseous mixture. The diffusion of dissolved air in water is, however, neglected.

$$
\begin{aligned}
& \underbrace{\frac{\partial \rho_{\mathrm{a}} n S_{\mathrm{r}, \mathrm{g}}}{\partial t}+\operatorname{div}\left(\underline{i}_{\mathrm{a}}+\rho_{\mathrm{a}} \underline{f}_{\mathrm{g}}\right)}_{\text {Dry air in gas phase }} \\
& +\underbrace{\frac{\partial H \rho_{\mathrm{a}} n S_{\mathrm{r}, \mathrm{w}}}{\partial t}+\operatorname{div}\left(\rho_{\mathrm{a}} H f_{-\mathrm{w}}\right)}_{\text {Dissolved air in water }}=0
\end{aligned}
$$

where $\underline{i}_{\mathrm{a}}$ is the non-advective flux of dry air, $\rho_{\mathrm{a}}$ is dry air density and $H$ is Henry's coefficient.

Henry's coefficient $H$ allows determining the dissolved air volume in liquid water. The dissolved air mass is supposed to be sufficiently low that water properties are not influenced.

\subsubsection{Diffusion of dry air}

The dry air diffusion flow is related to dry air density gradient. Using the diffusion theory adapted to 
porous medium, the non-advective dry air flows can be computed by:

$\underline{i}_{\mathrm{a}}=-D_{\mathrm{atm}} \tau_{\mathrm{v}} n S_{\mathrm{r}, \mathrm{g}} \underline{\operatorname{grad}}\left(\rho_{\mathrm{a}}\right)$

\subsubsection{Motion of gas}

The generalised Darcy's law for multiphase medium gives the gas velocity:

$f_{-\mathrm{g}}=-\frac{k_{\text {int }} k_{\mathrm{r}, \mathrm{g}}}{\mu_{\mathrm{g}}}\left[\underline{\nabla} p_{\mathrm{g}}+g \rho_{\mathrm{g}} \underline{\nabla} y\right]$

where $\mu_{\mathrm{g}}$ is the gas dynamic viscosity, $k_{\mathrm{r}, \mathrm{g}}$ is the gas relative permeability and $\rho_{\mathrm{g}}$ is the gas density.

The gas permeability varies in non-saturated conditions and is a decreasing function of the water saturation.

\subsection{Heat diffusion}

In this model of heat transfer in non-saturated medium, the different components are in a thermal equilibrium. A unique temperature is defined for the medium $\left(T_{\mathrm{s}}=T_{\mathrm{w}}=T_{\mathrm{a}}=T_{\mathrm{v}}=T_{\mathrm{da}}\right)$ and only one balance equation of energy is necessary.

\subsubsection{Conservation of the heat}

Neglecting kinetic energy and pressure energy terms, enthalpy balance equation is written as:

$$
\frac{\partial \phi}{\partial t}+L \dot{E}_{\mathrm{H}_{2} \mathrm{O}}^{\mathrm{w} \rightarrow \mathrm{v}}+\operatorname{div}(\underline{q})-Q=0
$$

where $\phi$ is the enthalpy of the medium, $L$ is the latent heat of water vaporisation, $\dot{E}_{H_{2}}^{w} \vec{O}^{v}$ is the rate of water evaporation, $q$ is the heat flow and $Q$ is a volume heat source.

Evaporation rate may be evaluated thanks to water vapour balance Eq. (12) and enthalpy balance equation becomes (Eq. (13)):

$\frac{\partial \rho_{\mathrm{v}} n S_{\mathrm{r}, \mathrm{g}}}{\partial t}+\operatorname{div}\left(\underline{i}_{\mathrm{v}}+\rho_{\mathrm{v}} f_{-\mathrm{g}}\right)=\dot{E}_{\mathrm{H}_{2} \mathrm{O}}^{\mathrm{w} \rightarrow \mathrm{v}}$

$$
\begin{aligned}
& \underbrace{\frac{\partial \phi}{\partial t}+L \frac{\partial \rho_{\mathrm{v}} n S_{\mathrm{r}, \mathrm{g}}}{\partial t}}_{\text {Heat storage }} \\
& +\underbrace{\operatorname{div}(\underline{q})+L \operatorname{div}\left(\underline{i}_{\mathrm{v}}+\rho_{\mathrm{v}} f_{-\mathrm{g}}\right)}_{\text {Heat transfer }}-Q=0
\end{aligned}
$$

Eq. (13) can be re-written as following:

$\frac{\partial \phi^{\prime}}{\partial t}+\operatorname{div}\left(\underline{q}^{\prime}\right)-Q=0$

where $\phi^{\prime}$ and $q^{\prime}$ are obtained by adding the contribution of water vaporisation to $\phi$ and $\underline{q}$.

\subsubsection{Quantity of heat storage: enthalpy}

The enthalpy of the system is given by the sum of each component's enthalpy. The contribution of dissolved air is only neglected.

$$
\begin{aligned}
\phi^{\prime}= & n S_{\mathrm{r}, \mathrm{w}} \rho_{\mathrm{w}} c_{\mathrm{p}, \mathrm{w}}\left(T-T_{0}\right)+n S_{\mathrm{r}, \mathrm{g}} \rho_{\mathrm{a}} c_{\mathrm{p}, \mathrm{a}}\left(T-T_{0}\right) \\
& +(1-n) \rho_{\mathrm{s}} c_{\mathrm{p}, \mathrm{s}}\left(T-T_{0}\right) \\
& +n S_{\mathrm{r}, \mathrm{g}} \rho_{\mathrm{v}} c_{\mathrm{p}, \mathrm{v}}\left(T-T_{0}\right)+n S_{\mathrm{r}, \mathrm{g}} \rho_{\mathrm{v}} L
\end{aligned}
$$

where $c_{\mathrm{p}, \alpha}$ is the specific heat of the component $\alpha$.

The last enthalpy term corresponds to the heat stored during the water vaporisation. Latent heat $L$ depends on temperature but this property is considered as constant in the model.

\subsubsection{Heat transport}

The heat transport is related to three effects: conduction, convection by the fluids and vaporisation.

$$
\begin{aligned}
\underline{q}^{\prime}= & -\Gamma \underline{\nabla} T+\left(c_{\mathrm{p}, \mathrm{w}} \rho_{\mathrm{w}} f_{-\mathrm{w}}+c_{\mathrm{p}, \mathrm{a}}\left(\underline{i}_{\mathrm{a}}+\rho_{\mathrm{a}} f_{-\mathrm{g}}\right)\right. \\
& \left.+c_{\mathrm{p}, \mathrm{v}}\left(\underline{i}_{\mathrm{v}}+\rho_{\mathrm{v}} f_{-\mathrm{g}}\right)\right)\left(T-T_{0}\right) \\
& +\left(\underline{i}_{\mathrm{v}}+\rho_{\mathrm{v}} f_{-\mathrm{g}}\right) L
\end{aligned}
$$

where $\Gamma$ is the medium conductivity.

The thermal conductivity of the medium depends on thermal properties of the components. Different expressions can be defined based on simplified models or determined thanks to some experiment results.

Some authors explicitly model also the solid convection, which is typically a large-strains, large-displacements effect. Our model takes the large strains and large rotations of the sample into account, thanks to a Lagrangian actualised formulation (Charlier, 1987). Therefore, the equilibrium and balance equations, as well as the water, air and heat flows are expressed in the moving current configuration. This implies that the solid convection effect is implicitly taken into account. 


\subsubsection{Couplings}

In very impervious medium, the main heat transfer is conduction, which depends on the thermal conductivity and temperature gradient. Fluids in pores influence the conductivity and create thus some coupling between fluid and heat transfer. The other principal coupling effect results from convection: water, vapour and air transport a quantity of heat. Moreover, vaporisation of water needs energy, which influences enthalpy balance equation.

\section{Mechanical behaviour modelling}

In a clay barrier, saturation and suction can vary considerably. Research experiments have shown that suction has a strong influence on the mechanical properties (Alonso and Al, 1990; Delage, 1987): stiffness and shear strength of the soil increase with suction; swelling or collapse can be induced; some irreversible deformations can even take place...

The mechanical behaviour modelling should be able to take this suction effect into account when the soil undergoes desaturation processes.

\subsection{Stress state variables}

The choice of stress state variables to describe the stress-strain relation is still an open question. Many researchers (Bishop and Blight, 1963) have attempted to incorporate the suction $s$ explicitly into an effective stress expression. For example, Bishop's postulate:

$\sigma_{i j}^{\prime}=\sigma_{i j}-p_{g} \delta_{i j}+\chi\left(p_{g}-p_{w}\right) \delta_{i j}$

where $\sigma_{i j}^{\prime}$ is the effective stress tensor, $\sigma_{i j}$ is the total stress tensor, $\chi$ represents the Bishop's coefficient which is a function of the saturation $S_{\mathrm{r}, \mathrm{w}}, \delta_{i j}$ refers to the Kronecker's tensor.

This concept presents some advantages: it is easy to implement into a finite element code (Schrefler et al., 1990; Charlier and Radu, 1997); it provides qualitatively good predictions for problems involving mainly shear stresses...

But its application to modelling of mechanical behaviour of unsaturated soils is limited mainly because of the following reasons.

(1) Generally, the volumetric behaviour cannot be properly modelled with this postulate. In particular, it is unable to model the collapse behaviour, which is a typical phenomenon of unsaturated soils during the wetting phase under certain external charges.

(2) The Bishop's coefficient $\chi$ is a very complicate function. Experimental investigations (Jennings and Burland, 1962) have shown that it may depend on the saturation $S_{\mathrm{r}, \mathrm{w}}$ but there is non-unique relation $\chi-S_{\mathrm{r}, \mathrm{w}}$ for a given soil sample with different void ratios.

(3) The value of the $\chi$ is stress path dependent.

(4) The experimental determination of $\chi$ is very difficult.

However, a simplified Bishop's postulate with $\chi=S_{\mathrm{r}, \mathrm{w}}$ can be used to model problems where the shear strain is dominant. It can be associated to any classical model (e.g. Drucker-Prager's model, see Charlier et al., 1997). This is the classical efficiency of an effective stress concept, which condensates here the suction effect into a single Eq. (17).

All these considerations lead to use two independent stresses state variables to model the mechanical behaviour of unsaturated soils. That is:

the net stresses tensor: $\sigma_{i j}^{*}=\sigma_{i j}-p_{\mathrm{g}} \delta_{i j}$

the suction : $\quad s=p_{\mathrm{g}}-p_{\mathrm{w}}$

It is proved to be suitable for the modelling of the mechanical behaviour of unsaturated soils from the theoretical as well as the experimental points of view (Fredlund and Morgenstern, 1977), and it allows to models efficiently the swelling-collapse behaviour of compacted clays. Now the soil mechanics constitutive model is a function of the two independent stress state variable, which affects nonlinearly the elastic behaviour, the yield surface shape, and the hardening function. A new constitutive model has to be fully developed, and cannot be simply an extension of a previous model developed for a saturated soil.

\subsection{Alonso-Gens' mechanical model}

The model proposed by Alonso et al. (1990) is based on the well-known CamClay model. It is written within the framework of the independent stresses state variables defined here above. In the numerical model, the plastic yield surfaces are written in a three-dimensional stress space: $I_{\sigma}^{*}-I I_{\hat{\sigma}}^{*}-S$, where $I_{\sigma}^{*}$ is the first net stress invariant and $I I_{\hat{\sigma}}^{*}$ refers to the second net deviatoric stress invariant. 


\subsubsection{The yield surfaces}

In the $I_{\sigma}^{*}-I I_{\hat{\sigma}}^{*}$ space, the yield surface of Alonso's model can be written for a given value of suction as:

$F_{1} \equiv\left(I_{\sigma}^{* 2}+\left(I_{0}-P_{\mathrm{s}}\right) I_{\sigma}^{*}-I_{0} P_{\mathrm{s}}\right) \bar{r}^{2}+I I_{\hat{\sigma}}^{* 2}=0$

where $\bar{r}$ is defined as a reduced radius, $I_{0}$ is the preconsolidation pressure of the soil and $P_{\mathrm{s}}$ represents the soil strength in extension.

Reduced radius represents the failure states and is given by the ratio of the second and first stress invariant. It can depend or not on the Lode's angle $\alpha$, according to the chosen formulation (Fig. 1):

$\bar{r}=\left\{\begin{array}{cc}C^{t e} & \text { Von }- \text { Mises } \\ a(1+b \sin 3 \alpha)^{n} & \text { Van - Eekelen }\end{array}\right.$

where $a, b, n$ are constants that are linked to the internal friction angles in compression and extension; they may vary with the suction.

The soil strength in extension $P_{\mathrm{s}}$ is an increasing function of suction. The following expression is used:

$P_{\mathrm{s}}=3 c / \operatorname{tg} \phi_{\mathrm{c}}$

where $c$ is the cohesion and $\phi_{\mathrm{c}}$ refers to the internal friction angle in compression.

Both characteristics $c$ and $\phi_{\mathrm{c}}$ may vary with suction and functions $c(s)$ and $\phi_{\mathrm{c}}(s)$ based on experimental results can be introduced into the code.

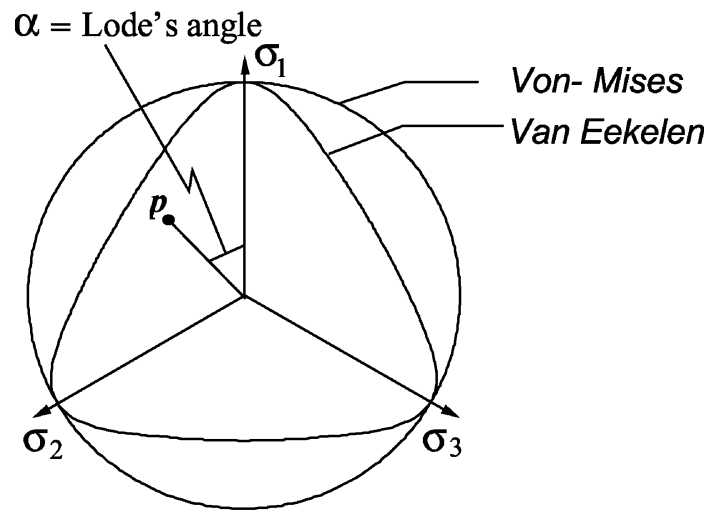

Fig. 1. Lode's angle dependence of the yield surface.

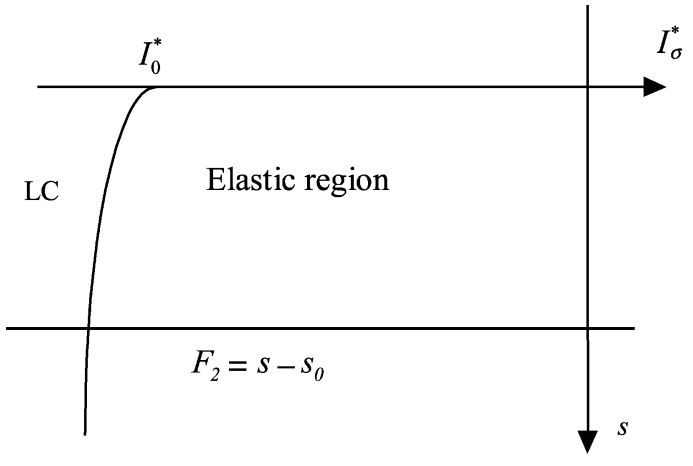

Fig. 2. Yield surface in the $I_{\sigma}^{*}-s$ plane.

Pre-consolidation pressure $I_{0}$ varies with the suction (Fig. 2). Alonso et al. (1990) proposed the following relation:

$I_{0}=p_{\mathrm{c}}\left(\frac{I_{0}^{*}}{p_{\mathrm{c}}}\right)^{\frac{\lambda(0)-\kappa}{\lambda(s)-\kappa}}$

where $I_{0}^{*}$ represents the pre-consolidation pressure in saturated condition, $p_{\mathrm{c}}$ is a reference pressure, $\kappa$ is the elastic slope of the compressibility curve against the net mean stress and $\lambda(s)$ refers to the plastic slope of the compressibility curve against the net mean stress.

Suction contributes to stiffening the soil against the external loads, which means that compressibility index $\lambda(s)$ varies with the suction according to:

$\lambda(s)=\lambda(0)[(1-r) \exp (-\beta s)+r]$

where $\lambda(0)$ is the plastic slope for the saturated condition; $r$ and $\beta$ are parameters describing the changes in soil stiffness with suction.

The trace of the pre-consolidation pressure in the plane (Eq. (22)) defines another part of the yield surface called loading collapse (LC) used for modelling the collapse behaviour under wetting.

Irreversible volumetric deformations may be induced by variations of suction. Therefore, a second yield surface $(S I)$ defined in the $I_{\sigma}^{*}-S$ plane is used (Fig. 2):

$F_{2} \equiv s-s_{0}=0$

where $s_{0}$ is a yield value, which represents the maximum suction submitted to the soil. 


\subsubsection{Responses of the model}

Total strains of the soil are induced by variations of stresses, suction and temperature. Stresses and suction can produce plastic deformations but thermal strains are assumed to be reversible. Total strains are defined as the addition of different contributions:

$\dot{\varepsilon}_{k l}=\dot{\varepsilon}_{k l-\mathrm{m}}^{\mathrm{e}}+\dot{\varepsilon}_{k l-\mathrm{m}}^{\mathrm{p}}+\dot{\varepsilon}_{k l-\mathrm{s}}^{\mathrm{e}}+\dot{\varepsilon}_{k l-\mathrm{s}}^{p}+\dot{\varepsilon}_{k l-\mathrm{T}}^{\mathrm{e}}$

where subscripts $\mathrm{m}, \mathrm{s}$ and $\mathrm{T}$ are related respectively to mechanical, suction and thermal contributions.

The elastic and plastic strains due to stress changes (mechanical solicitations) are defined by:

$\dot{\varepsilon}_{k l-\mathrm{m}}^{\mathrm{e}}=\left(C_{i j k l}^{\mathrm{e}}\right)^{-1} \dot{\sigma}_{i j}^{\mathrm{e}}$ (Elastic deformations)

$\dot{\varepsilon}_{k l-\mathrm{m}}^{\mathrm{p}}=\dot{\lambda}^{\mathrm{p}} \frac{\partial G}{\partial \sigma_{k l}}$ (Plastic deformations)

where $C_{i j k l}^{e}$ is the Hooke's tensor, $\dot{\sigma}_{i j}^{\mathrm{e}}$ is the elastic net stress tensor, $Q$ is the symbol for the plastic potential surface and $i^{p}$ is obtained by the consistency condition. of:

A non-linear elasticity can be considered by means

$$
\begin{aligned}
& K=\frac{1+e}{3 \kappa} I_{\sigma}^{*} \\
& G=\frac{3(1-2 v)}{2(1+v)} K
\end{aligned}
$$

where $K$ is the soil bulk modulus, $G$ is the shear modulus, $v$ is the Poisson's coefficient and $e$ is the void ratio.

A non-associated flow rule in the $I_{\sigma}^{*}-I I_{\sigma}^{*}$ plane can be introduced into the model. The following equation is used in order to obtain zero lateral strain for stress states corresponding to Jaky's $K_{0}$ values:

$$
\frac{\partial Q}{\partial I_{\hat{\sigma}}^{*}}=\eta \frac{\partial F}{\partial I_{\hat{\sigma}}^{*}}
$$

where $\eta$ is a parameter related to the $\bar{r}, \kappa$ and $\lambda(s)$.

The deformations induced by suction changes (hydric path) are:

$\dot{\varepsilon}_{k l-\mathrm{s}}^{\mathrm{e}}=h^{\mathrm{e}} \dot{s} \delta_{k l}$ (Elastic deformations)

$$
\dot{\varepsilon}_{k l-\mathrm{s}}^{p}=h^{p} \dot{s} \delta_{k l} \text { (Plastic deformations) }
$$

with

$$
\begin{aligned}
& h^{\mathrm{e}}=\frac{\kappa_{\mathrm{s}}}{3(1+e)\left(s+P_{\mathrm{at}}\right)} \\
& h^{p}=\frac{\lambda_{s}-\kappa_{s}}{3(1+e)\left(s+P_{a t}\right)}
\end{aligned}
$$

where $\lambda_{\mathrm{s}}$ and $\kappa_{\mathrm{s}}$ are stiffness parameters for changes in suction and $P_{\text {at }}$ is the atmospheric pressure. It should be noted that $\lambda_{\mathrm{s}}$ and $\kappa_{\mathrm{s}}$ could vary with the stress level.

The plastic compaction related to suction increase takes place when the suction is larger than $s_{0}$.

The elastic thermal dilatation is introduced in the model by:

$\dot{\varepsilon}_{k l-\mathrm{T}}^{\mathrm{e}}=\xi \dot{T} \delta_{k l}$

where $\xi$ is the dilatation coefficient.

The evolution of yield surfaces is controlled by the total plastic volumetric strain $\varepsilon_{v}^{\mathrm{p}}$ created in the soil. Two hardening laws define the evolution of state variables $I_{0}^{*}$ and $s_{0}$ with the irreversible strain:

$\mathrm{d} I_{0}^{*}=\frac{(1+e) I_{0}^{*}}{\lambda(0)-\kappa} \mathrm{d} \varepsilon_{\mathrm{v}}^{\mathrm{p}}$

$\mathrm{d} s_{0}=\frac{(1+e)\left(s_{0}+P_{\mathrm{at}}\right)}{\lambda_{\mathrm{s}}-\kappa_{\mathrm{s}}} \mathrm{d} \varepsilon_{\mathrm{v}}^{\mathrm{p}}$

Table 1 lists the parameters of the mechanical model and their determination means. After appropri-

Table 1

Determination means of parameters

\begin{tabular}{ll}
\hline Parameters & Determination means \\
\hline$e_{0}$ & Measured \\
$\lambda(0)$ & Oedometer loading-unloading test in saturated state \\
$I_{0}^{*}$ & Id. \\
$\lambda_{\mathrm{s}}$ & $\begin{array}{l}\text { Oedometer or isotropic wetting-drying tests under } \\
\text { different external charges }\end{array}$ \\
$\kappa_{\mathrm{s}}$ & Id. \\
$r$ & Series of suction-controlled oedometer tests \\
$\beta$ & Id. \\
$p_{\mathrm{c}}$ & Id. The measurements of $I_{0}$ at different level of \\
$\kappa$ & suction are required to calibrate $p_{\mathrm{c}}$ \\
$C(s)$ & Id. The function $\kappa(s)$ may be observed \\
$\phi_{\mathrm{c}}(s)$ & Series of suction-controlled triaxial tests \\
\hline & Id. \\
\hline
\end{tabular}


ate manipulations, the general constitutive relationship in reverse form can be written as:

$\dot{\sigma}_{i j}=D_{i j k l} \dot{\varepsilon}_{k l}-V_{i j} \dot{s}-R_{i j} \dot{T}$

where $D_{i j k l}$ is the classical elasto-plastic tensor, $V_{i j}$ is a tensor related to suction and $R_{i j}$ refers to thermal effects.

For the integration of the constitutive relation, we have used the so-called $\theta$ point method. To obtain more accurate results, the integration time step $\Delta t$ is divided into $N$ sub-steps $\mathrm{d} t$. The sub-steps size can be automatically adjusted in function of the strain increment $\|\Delta \varepsilon\|$ or chosen by the user. For each sub-time step $\mathrm{d} t$, the integration of Eq. (37) can be expressed as:

$\sigma_{i j}(N+\theta \mathrm{d} t)=\sigma_{i j}(N)+\dot{\sigma}_{i j}(N) \theta \mathrm{d} t$

$\sigma_{i j}(N+1)=\sigma_{i j}(N)+\dot{\sigma}_{i j}(N+\theta \mathrm{d} t) \mathrm{d} t$

where $N$ denotes the sub-time step number, $\theta$ is a numerical parameter that takes the value between 0 and 1 (usually $\theta>0.5$ for reasons of numerical stability).

The hardening variables can be also integrated in the same way. This version of the model can simulate the swelling and collapse behaviours but has some limitations for highly expansive materials: the plastic swelling deformation cannot be taken into account.

\section{Finite element formulation}

A bi-dimensional large strain finite element has been implemented in the finite element code LAGAMINE, developed at Liege University for 20 years (Charlier, 1987). That element possesses five degrees of freedom at each node: two displacements of the soil skeleton, a liquid water pressure, a gas (dry air + vapour) pressure and a temperature. The number of nodes is variable (three, four, six or eight) and the element is isoparametric, that is to say that the coordinates, velocities, pore pressure (water and gas) and temperature are discretised by the same shape functions.

That element has a particularity: if the description of the co-ordinates are parabolic, the spatial variation of the strains (spatial derivatives of the velocities) are thus linear, and in the same way the stresses (in elasticity). On the other hand, the variations of pore pressures and the temperature are parabolic, that is to say one degree higher than stresses. In some cases, that difference degree between stresses and pore pressure or temperature can provide numerical results less accurate. That is why, with that element, we have introduced the possibility to have a parabolic discretisation for the co-ordinates and the mechanics at the same time than a linear discretisation for the fluid (pore pressures and the temperature).

The so-called stiffness matrix is the basic tool that allows to numerically converge to an accurate state, that is, a state that respects the balance equations (1), (8) and (13) and the solid mechanics equilibrium. Thanks to the space discretisation, these equations are transformed into nodal values $\underline{F}$ (fluxes and forces) whose internal and external values are to be equal. If not, a new solution, that is, a new approximation of nodal fluid pressure, temperature and displacements has to be determined. Symbolically, the stiffness matrix $\underline{\underline{K}}$ is obtained thanks to first-order Taylor development of the nodal values:

$\underline{F}^{i+1}=\underline{F}^{i}+\frac{\partial \underline{F}}{\partial \underline{x}} \underline{\Delta x}=\underline{F^{i}}+\underline{\underline{K}} \underline{\Delta x}$

A quadratic convergence may be obtained if and only if the stiffness matrix $\underline{K}$ is accurately computed. For this purpose, the thermo-hydro-mechanical coupling matrix is here a monolithical one. The stiffness matrix in the Newton-Raphson sense of the coupled element is completely modified with regard to those corresponding to uncoupled elements. Indeed, for example concerning the coupling between mechanic and water pressure, we have to compute not only the displacements effect on nodal forces and the water pressure on nodal water flows, but also the water pressure effect on nodal forces (by way of the Terzaghi's postulate) and the displacements effects on nodal water flows (by way of the storage). Finally, in great transformations, we have to compute the effect of geometrical modifications on nodal water, gas and thermal flows. All coupling terms of the matrix are taken into account, providing a good convergence rate for most treated problems.

This stiffness matrix associates then five degrees of freedom per node and can be schematised as follows (Fig. 3): where $\underline{F}_{\mathrm{M}}$ represents the nodal mechanical force, $\underline{F}_{\mathrm{W}}$ the nodal water flux, $\underline{F}_{\mathrm{G}}$ the nodal gas flux, 


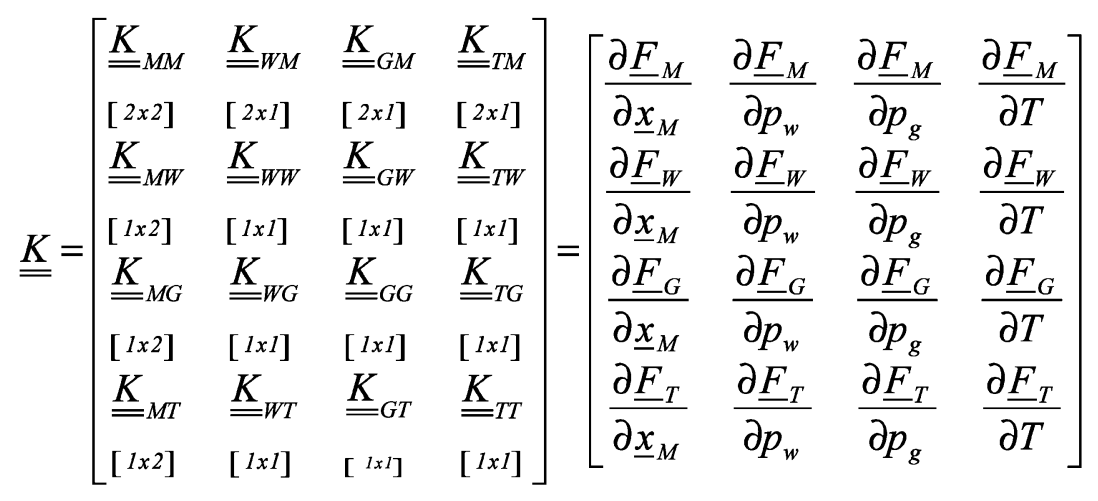

Fig. 3. Stiffness matrix.

$\underline{F}_{\mathrm{T}}$ the nodal thermal flux, $\underline{x}_{\mathrm{M}}$ the nodal displacements, $p_{\mathrm{w}}$ the nodal water pressure, $p_{\mathrm{g}}$ the nodal gas pressure and $T$ the nodal temperature.

The four mechanical, water flow, gas flow and thermal sub-matrixes, $\underline{\underline{K}}_{\mathrm{MM}}, \underline{\underline{K}}_{\mathrm{WW}}, \underline{\underline{K}}_{\mathrm{GG}}$ and $\underline{\underline{K}}_{\mathrm{TT}}$ are classical. All the other sub-matrixes are coupling matrixes. For example, the two coupling sub-matrixes $\underline{K}_{\mathrm{MW}}$ and $\underline{K}_{\mathrm{WM}}$ represent respectively the coupling of mechanics onto water flow and water flow onto mechanics: they are obtained thanks to the derivation of the water nodal fluxes with respect to the mechanical displacements, and to the derivation of the mechanical nodal forces with respect to the nodal water pore pressures. The contribution of all theses coupling sub-matrixes is of first importance to obtain a good convergence, while their numerical evaluation is quite simple to perform.

Let us note also that to increase the numerical stability, nodal water flows, gas flows and thermal flows, and the corresponding stiffness, sub-matrixes are always computed in the initial configuration.

\section{Validation test}

The following modelling has been performed in the framework of a European Community research project entitled Calculation and testing of behaviour of unsaturated clay (Catsius clay), to investigate both temperature and artificial hydration effects on the deformation and moisture transfer in the soil. The results of a small-scale wetting-heating test performed on highly compacted bentonite have been available (CIEMAT Report, 1994). The test has been performed inside a thermohydraulic cell, which is schematised in Fig. 4. The cell case was a cylinder made of stainless steel, with an inner diameter of 15.0 $\mathrm{cm}$, an inner height of $15.6 \mathrm{~cm}$ and a thickness of 3.6 $\mathrm{cm}$. The heater, placed in the upper part of the cell and along its axis, was a cylinder with a height of $10.0 \mathrm{~cm}$ and a diameter of $2.0 \mathrm{~cm}$. It consisted of a resistance electronically regulated by an $\mathrm{ON} / \mathrm{OFF}$ temperature controller with a thermocouple sensor. The porous plate, placed in the lower part of the cell, was a cylinder with a height of $1.0 \mathrm{~cm}$ and a diameter of $15.0 \mathrm{~cm}$. It was connected through the hydration ports to an automatic pump.

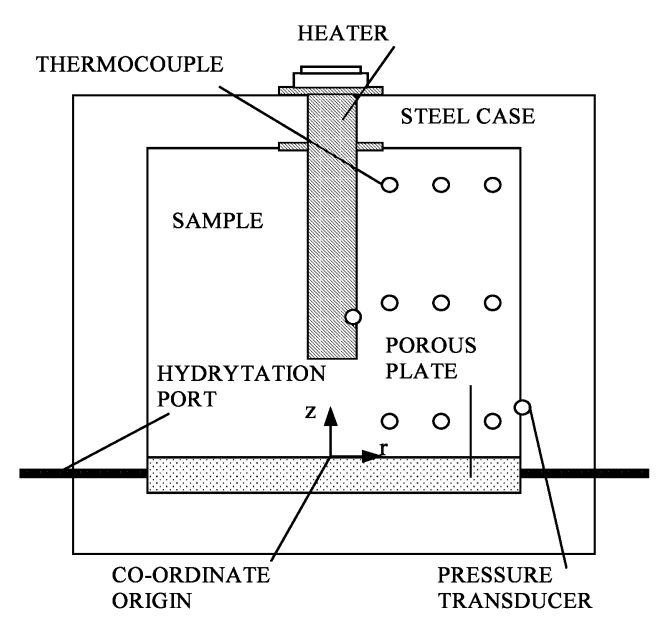

Fig. 4. Configuration of the thermohydraulic cell. 
The test was carried out by applying a constant heating power of $40 \mathrm{~W}$ and a constant water pressure of $1.1 \mathrm{MPa}$ to the lower porous plate during the time the experience elapsed. The outer cell surface was in contact with the ambient air of the laboratory. The total duration of the experience was $2401.6 \mathrm{~h}$.

During the test, temperatures at different points (nine thermocouples), water volume intake and swelling pressure generated (one pressure transducer) have been measured. The outer cell surface has been in contact with ambient air.

\subsection{Hydraulic and thermal properties}

The compacted bentonite used (Spanish montmorillonite from Almería) had initially $49 \%$ water saturation and a porosity of $40 \%$. Measured data of water content in function of the suction were available (CIEMAT Report, 1994; UPC Report, 1996) for this clay. The chosen capillary pressure expression defines water saturation as a function of suction:

$$
S_{\mathrm{r}, \mathrm{W}}=S_{\mathrm{r}, \mathrm{res}}+C S W 3 \frac{\left(S_{\mathrm{r}, \mathrm{u}}-S_{\mathrm{r}, \mathrm{res}}\right)}{C S W 3+(C S W 1 . s)^{C S W 2}}
$$

where $S_{\mathrm{r}, \mathrm{u}}$ is the maximum saturation in the soil and $S_{\mathrm{r}, \text { res }}$ is the residual saturation for a very high value of suction. Values of $S_{\mathrm{r}, \mathrm{u}}$ and $S_{\mathrm{r}, \mathrm{res}}$ found experimentally are respectively 1.0 and 0.1 .

Calibration of this function on measured data gives the following values of parameters: $C S W 1=3.5 \times 10^{6}$ $\mathrm{Pa}^{-1}, C S W 2=0.90$ and $C S W 3=120$.

Relative permeability curves are based on works of Brooks and Corey (1964):

$k_{\mathrm{r}, \mathrm{w}}=S_{\mathrm{e}}^{3}=\left(\frac{S_{\mathrm{r}, \mathrm{w}}-S_{\mathrm{r}, \mathrm{ess}}}{S_{\mathrm{r}, \mathrm{u}}-S_{\mathrm{r}, \mathrm{res}}}\right)^{3}$

$k_{r, g}=\left(1-S_{e}\right)^{2}\left(1-S_{e}^{\frac{5}{3}}\right)$

where $S_{\mathrm{e}}$ the effective saturation.

The water retention curve and the permeability are found to have an important influence on the water intake volume and the final saturation degree. The intrinsic permeability $k_{\text {int }}=4.7 \times 10^{-21} \mathrm{~m}^{2}$ was chosen in order to reproduce the experimental water intake curve.
Table 2

Parameters of the flow model

\begin{tabular}{llll}
\hline Parameters & Symbol & Value & Unit \\
\hline Grains density & $\rho_{\mathrm{s}}$ & $2.75 \times 10^{3}$ & $\mathrm{~kg} \cdot \mathrm{m}^{-3}$ \\
Grains specific heat & $c_{\mathrm{p}, \mathrm{s}}$ & $8.79 \times 10^{2}$ & $\mathrm{~J} \cdot \mathrm{kg}^{-1} \mathrm{~K}^{-1}$ \\
Water density & $\rho_{\mathrm{w}}$ & $1.00 \times 10^{3}$ & $\mathrm{~kg} \cdot \mathrm{m}^{-3}$ \\
Water dynamic viscosity & $\mu_{\mathrm{w}}$ & $1.009 \times 10^{-3}$ & $\mathrm{~Pa} \cdot \mathrm{s}$ \\
Water specific heat & $c_{\mathrm{p}, \mathrm{w}}$ & $4.180 \times 10^{3}$ & $\mathrm{~J} \cdot \mathrm{kg}^{-1} \mathrm{~K}^{-1}$ \\
Air density & $\rho_{\mathrm{a} 0}$ & 1.205 & $\mathrm{~kg} \cdot \mathrm{m}^{-3}$ \\
Air dynamic viscosity & $\mu_{\mathrm{a}}$ & $1.80 \times 10^{-5}$ & $\mathrm{~Pa} \cdot \mathrm{s}$ \\
Air specific heat & $c_{\mathrm{p}, \mathrm{a}}$ & $1.00 \times 10^{3}$ & $\mathrm{~J} \cdot \mathrm{kg}^{-1} \mathrm{~K}^{-1}$ \\
Water vapour specific heat & $c_{\mathrm{p}, \mathrm{v}}$ & $1.90 \times 10^{3}$ & $\mathrm{~J} \cdot \mathrm{kg}^{-1} \mathrm{~K}^{-1}$ \\
Latent heat of vaporisation & $L$ & $2.50 \times 10^{6}$ & $\mathrm{~J} . \mathrm{kg}^{-1}$ \\
Tortuosity & $\tau$ & 0.1 & - \\
\hline
\end{tabular}

The soil conductivity is a function of the saturation degree. The following relation was calibrated on experimental results (CIEMAT Report, 1994):

$\Gamma=1.0553 S_{\mathrm{r}, \mathrm{w}}+0.3573\left[\mathrm{~W} \mathrm{~m}^{-1} \mathrm{~K}^{-1}\right]$

The other parameters of the flow model are listed in Table 2, where common values of fluid properties have been chosen.

\subsection{Parameters related to the mechanical model}

The results of two series of suction controlled oedometer tests have been obtained to get the mechanical parameters (EUR Report, 1996). First one includes some tests with wetting-drying cycles under different constant vertical pressures, which allow the determination of $\lambda_{\mathrm{s}}, \kappa_{\mathrm{s}}$ and $s_{0}$ parameters (Eqs. (25), (33) and (34)). Another series of tests has been realised following several loading-unloading cycles under different constant suctions. Experimental values of $\lambda, \kappa$ and $p_{0}$ parameters have been found for different suctions.

In practice, determination of the parameters is sometimes difficult since experimental results are not always easily interpretable. For example, repeated tests do not every time reproduce the same results and it may be difficult to distinguish the transition between elastic and plastic parts.

Experiments show that elastic stiffness parameter $\kappa$ depends effectively on the suction imposed in the sample. However, in the present model, this value is assumed constant and an average value is chosen. Evolution of plastic stiffness parameter $\lambda$ shows a de- 


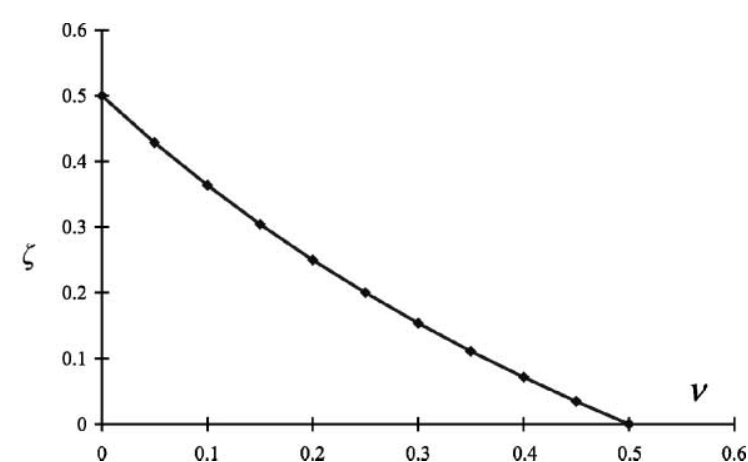

Fig. 5. Influence of $v$ on the shear module of soil.

crease with the suction. Eq. (24) is used to model the measured value.

Yield surface LC governed by Eq. (22) is very sensitive and its convexity is not always guaranteed. LC curve should model the evolution of $p_{0}$ with suction and depends on $\kappa, \lambda$ and $p_{\mathrm{c}}$ parameters. Two difficulties are often met during calibration: firstly, the condition $\lambda(s)>\kappa$ must be fulfilled in any cases and secondly, convexity of LC curve is linked with the value of $p_{\mathrm{c}}$, which is not easily determined directly from experimental results (a calibration procedure is used). LC calibration has, however, an important influence on the model responses.

Experimental values of elastic stiffness parameter $\kappa_{\mathrm{s}}$ for changes in suction depend on the stress state generated in the sample. This dependency is not taken into account in the model and a mean value has been chosen. Plastic stiffness parameter for changes in suction $\lambda_{\mathrm{s}}$ defines irreversible deformations related to suction increase. As far as the experiment follows

Table 3

Parameters of the mechanical model

\begin{tabular}{llll}
\hline Parameters & Symbol & Value & Unit \\
\hline Saturated virgin compression index & $\lambda(0)$ & 0.4041 & - \\
Elastic compression index & $\kappa$ & 0.015 & - \\
Saturated pre-consolidation pressure & $p_{0}^{*}$ & 0.6 & $\mathrm{MPa}$ \\
Elastic stiffness index upon suction & $\kappa_{\mathrm{s}}$ & 0.11 & - \\
Plastic stiffness index upon suction & $\lambda_{\mathrm{s}}$ & 0.25 & - \\
Maximum value of the suction & $s_{0}$ & 78.6 & $\mathrm{MPa}$ \\
Reference stress & $p_{\mathrm{c}}$ & 0.45 & $\mathrm{MPa}$ \\
Ratio $\lambda(s) / \lambda(0)$ for high suction & $r$ & 0.3 & - \\
Parameter to control the increase of & $\beta$ & 0.041 & $\mathrm{MPa}^{-1}$ \\
stiffness with suction & & & \\
\hline
\end{tabular}

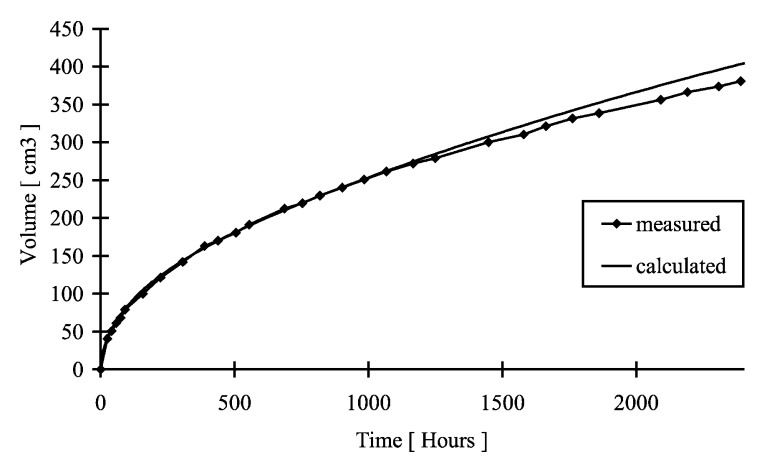

Fig. 6. Water intake evolution.

a wetting path, an average value of 0.25 is taken without looking into details at the experiment results.

As in this model, parameter $s_{0}$ refers to the maximum value of suction experienced by the soil; $s_{0}$ value is equal to the suction corresponding to the initial saturation value of sample before hydration. The initial suction in the sample has been chosen in the same way.

Friction angle and Poisson's ratio remains undefined after the exploitation of experimental results. Internal frictional angle controls the shape of the yield surface and the CSL. The adopted value of $\phi_{\mathrm{c}}$ is $35^{\circ}$. In the case of non-linear elasticity, the shear module $G$ depends on the Poisson's ratio $v$ and the stress level. From Eq. (28), we get:

$G=\frac{3(1-2 \nu)}{2(1+\nu)} \frac{(1+e)}{3 \kappa} I_{\sigma}^{*}=\zeta \frac{(1+e)}{\kappa} I_{\sigma}^{*}$

On Fig. 5, the proportional coefficient $\zeta$ is plotted in function of $v$. For a given value of stress state $I_{\sigma}^{*}$,

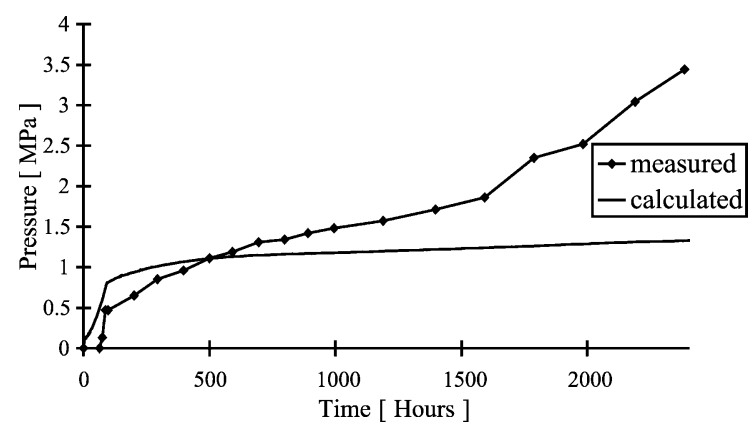

Fig. 7. Swelling pressure evolution. 


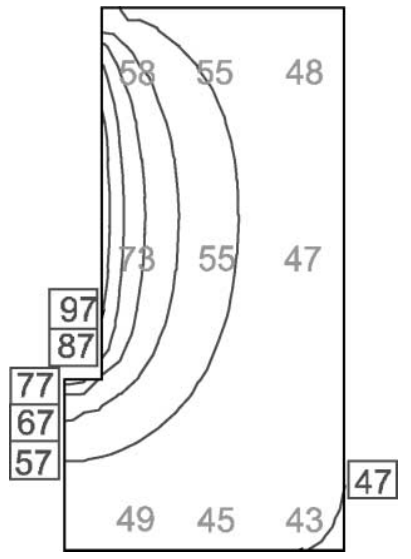

Fig. 8. Temperature field at the end of experience.

the shear module $G$ can decrease more than two times when $v$ changes from 0.3 to 0.4 . However, the shear modulus $G$ plays a most important role for generating deviatoric stresses. The chosen value of $v=0.4$ gives a satisfactory response. All the parameters used for the mechanical model are summarised in Table 3.

\subsection{Comparisons between simulation and experimen- tal results}

A 2D-axisymetric finite element simulation is realised with the help of the developed finite elements. The heating is modelled by imposing the temperature on the nodes of the sample in contact with the heater. The hydration procedure is modelled by increasing the water pressure on the nodes of porous plate. The

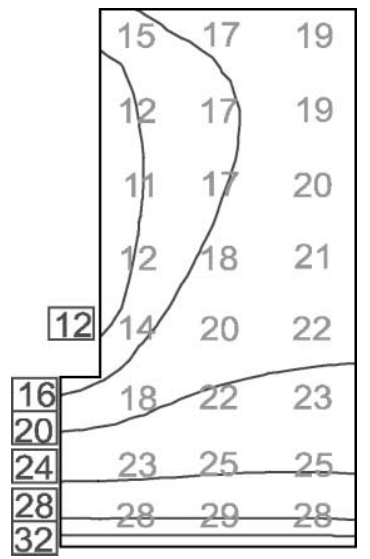

Fig. 9. Water content at the end of experience.
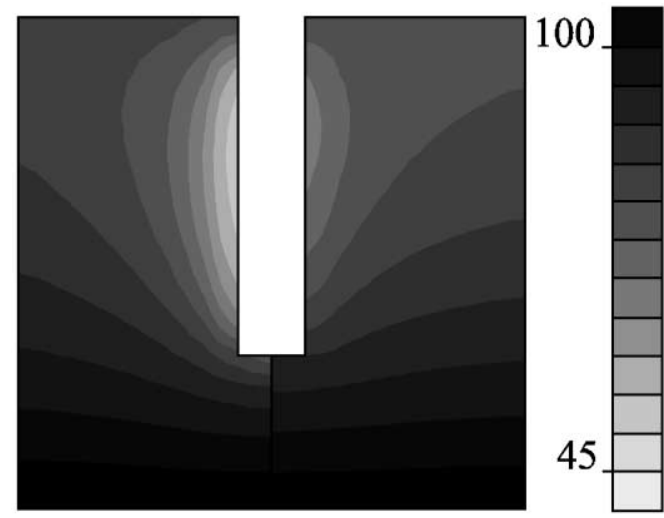

Fig. 10. Saturation (\%) at the end of the experiment.

convection transfer between the steel case and the ambient atmosphere is modelled thanks to frontier thermal elements.

The steel case is supposed to be impermeable to the water flows. Both steel case and porous plate deformations are neglected. The system is initially at ambient temperature $(293 \mathrm{~K})$. The gas pressure is supposed to remain fixed to the atmospheric pressure $(100 \mathrm{kPa})$. The initial saturation of the soil is $49 \%$ which gives an initial suction $s=78.6 \mathrm{MPa}$ according to the water retention curve. In the simulation, dissolved air will not be taken into account.

Fig. 6 shows water intake evolution with time. A very good result is obtained: the experimental and numerical curves are almost the same. The comparison between experimental and numerical result of the swelling pressure at the point with co-ordinates $r=7.5$
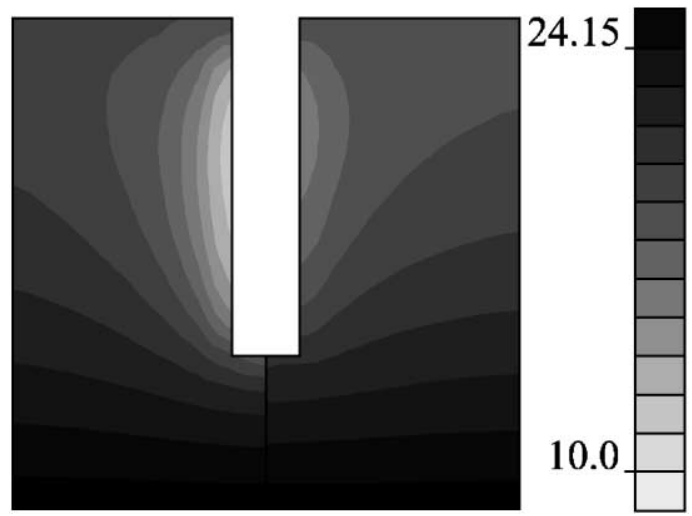

Fig. 11. Water content (\%) at the end of the experiment. 


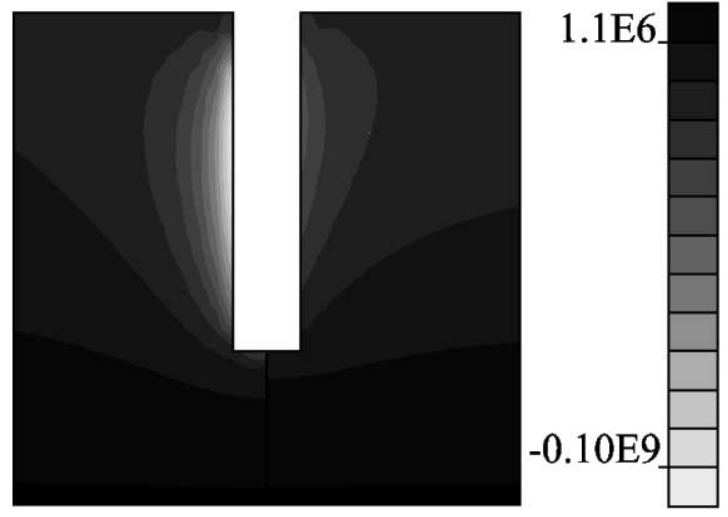

Fig. 12. Water pressure $(\mathrm{Pa})$ at the end of the experiment.

$\mathrm{cm}$ and $z=1.25 \mathrm{~cm}$ is presented in Fig. 7. The agreement is good at the beginning, but decreases at the end of experience. In fact, the model did not take into account some variations of certain parameters for this simulation, like $\kappa_{\mathrm{s}}$ varying with the net stress, $\kappa$ depending on the suction, etc... Moreover, only one experimental result of total pressure is available and measurement is located at a high stress gradient region. Results validity may be then questionable.

The calculated temperatures and water contents at the end of the experience are given in Figs. 8 and 9, respectively. The corresponding experimental measurements at some points are also presented in grey on the same figures. The calculated temperatures are a little higher than the experimental ones. The numerical water content seems to be slightly lower than the experimental one at the analysed points. But they are close to the experimental ones near the heater. The generation of water vapour near the heater is a crucial phenomenon to take here into account. The vapour flow depends deeply on the temperature field. The numerical results' quality is here a deep validation of the model.

All the results appear to be very sensitive to the retention curve, the relative and intrinsic permeability. One could also remark that soil deformations do not influence high water flows. Oppositely, the water flow has a deep influence on the mechanical behaviour.

The computations show the ability of the model to reproduce both mechanical behaviour and fluid experiment measurement. Final water content and temperature are close to the measured values. Moreover, the swelling pressure is qualitatively well predicted by the code.

\subsection{Influence of the gas pressure}

In the previous simulation, the gas pressure remains fixed to the atmospheric pressure. The (constant or variable) gas pressure effect has been studied thanks to simulations performed with (case A) and without (case B) a fixed gas pressure. In case B, the steel case is supposed impermeable to gas. For both cases, soil skeleton is supposed rigid.

On pictures 10-13, the left part is the result of case $\mathrm{A}$ and the right part is the one of case $\mathrm{B}$. The results show clearly differences between the two simulations. The saturation degree in case A varies from $47 \%$ to $100 \%$ while it varies from $62 \%$ to $100 \%$ in case B (Fig. 10). Water content (Fig. 11) and water pressure (Fig. 12) are consistent with the results of water saturation. In terms of suction, it means that the sample is submitted to a maximum suction of 85.1 MPa in case A and of $40.3 \mathrm{MPa}$ in case B (Fig. 13).

The computations have also shown that gas pressure increases in a range from 302 to $463 \mathrm{kPa}$. This gas pressure increase should also have an influence on mechanical behaviour. Indeed, this increase modifies suction field and net stresses, which are the independent variables of the mechanical model.

\subsection{Boundary condition and mass conservation}

In the chosen formulation, balance equations are written for each species. Water species is present in liquid phase (liquid water) and in gaseous phase (water vapour). Dry air is only present in the gaseous phase.

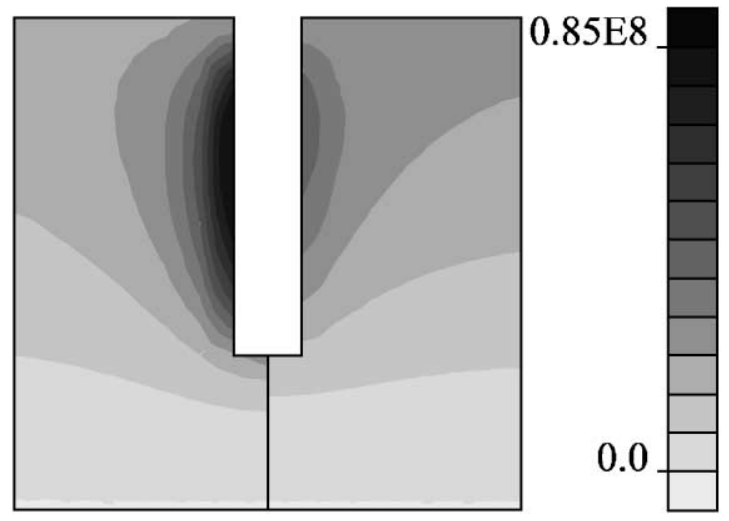

Fig. 13. Suction $(\mathrm{Pa})$ at the end of the experiment. 

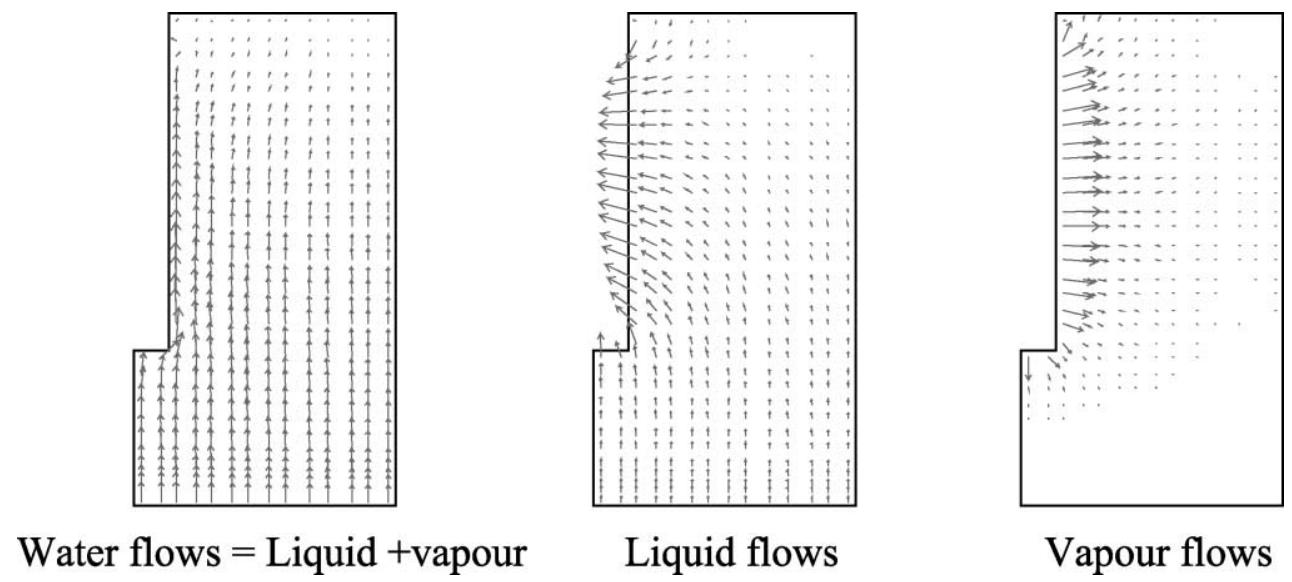

Fig. 14. Water flows at the end of the experiment.

On Fig. 13, global water flows, liquid water flows and water vapour flows are drawn. As balance equation of water species is used, only the global water flows respect the boundary condition: no flows are allowed through the boundary as it is considered as impermeable. However, liquid and vapour flows do not seem to respect the boundary condition: this is a drawback of the formulation.

Other formulations can be found with different combinations of degrees of freedom and balance equations. The different choices are listed in the Table 4.

If air pressure is a degree of freedom of the finite element, it will be impossible to impose gas pressure. This choice is not convenient for the type of problems dealt with. If balance equations of liquid and gaseous phases are written, only liquid and gas phase flows respect the boundary conditions. However, water vapour flows and dry air flows will not respect them. Moreover, in order to respect the global water mass

Table 4

Combination of degrees of freedom and balance equations

\begin{tabular}{|c|c|c|c|}
\hline DOF & Balance equation & $\begin{array}{l}\text { Boundary } \\
\text { conditions } \\
\text { respect }\end{array}$ & $\begin{array}{l}\text { Pressure } \\
\text { imposition }\end{array}$ \\
\hline$P_{\mathrm{w}}$ & Liquid + vapour & Water flow & Water pressure \\
\hline$P_{\mathrm{g}}$ & Dry air & Dry air flow & Gas pressure \\
\hline$P_{\mathrm{w}}$ & Liquid & Liquid water & Liquid pressure \\
\hline$P_{\mathrm{g}}$ & Dry air + vapour & Gas flow & Gas pressure \\
\hline$P_{\mathrm{w}}$ & Liquid + vapour & Water flow & Water pressure \\
\hline$P_{\mathrm{a}}$ & Dry air & Dry air flow & Dry air pressure \\
\hline$P_{\mathrm{w}}$ & Liquid & Liquid flow & Water pressure \\
\hline$P_{\mathrm{a}}$ & Dry air + vapour & Gas flow & Dry air pressure \\
\hline
\end{tabular}

conservation, some evaporation term must be computed and taken into account in the balance equation.

The origin of the problem is the following: water vapour characteristics (vapour pressure and vapour content) depend directly on temperature and water and gas pressures as quite equilibrium states are considered (Fig. 14). Thus, a water vapour balance equation is not necessary and a balance equation of all the components of the system cannot be written. So, one conservation equation corresponds to a mixture (liquid + vapour or dry air + vapour) and the boundary conditions will be respected by the mixture flows and not by the mixture's components flows.

This problem will need some further developments and is not solved in the present paper.

\section{Conclusion}

A complete theory of a thermo-hydro-mechanical coupling model for unsaturated soils is provided in this paper. A validation test is performed to show ability of the model to simulate the relevant phenomenon in nuclear waste storage. The numerical results well predict re-saturation of the sample near the porous plate and a dried area is created near the heater due to water evaporation. Final values of water content are close to experimental measurement. Comparison between numerical and experimental temperatures is very good; in this low-permeability soils, conduction is the main heat transfer mode. Moreover, the swelling pressure is qualitatively well predicted especially at the beginning 
of the experiment. Better results would be obtained if the variations of certain parameters were taken into account, like $\kappa_{\mathrm{s}}$ varying with the net stress, $\kappa$ depending on suction. Additional computations have shown the influence of the gas pressure. Final saturation and suction are lower if the variations of gas pressure are taken into account.

Eventually, the developed models are able to reproduce the mechanical behaviour of unsaturated clays and to predict moisture and air motions under thermal solicitations. They are helpful tools to study the nuclear waste repositories in deep clay formations.

Nevertheless these apparently very optimistic conclusions, the question of the theoretical and numerical model validation may be asked. Homogeneous and simple tests have been performed in order to ensure the accuracy and consistency of the model. However, only few analytical solutions are available to validate such development. Then modelling actual physical and mechanical tests is probably the only validation procedure presently available for THM clay barrier models. However, it is clear that the solution (in terms of parameter values for example) is probably never unique. Moreover, the number of experiments allowing material parameter calibration is high but is far from enough for a full determination, and some hypotheses have been assumed. Then the validation described in this paper is interesting in the sense that the proposed model has accurately reproduced most phenomena that have been accurately measured in the experiment.

\section{Acknowledgements}

The authors thank the Europe Community for the support through the research project Catsius clay project. The support from FNRS is also greatly acknowledged.

\section{References}

Alonso, E.E., Gens, A., Josa, A.A., 1990. A constitutive model for partly saturated soil. Géotechnique 40 (3), 405-430.

Bishop, A.W., Blight, G.E., 1963. Some aspects of effective stress in saturated and unsaturated soils. Géotechnique 3, 177-197.
Brooks, R.H., Corey, A.T., 1964. Hydraulic properties of porous media. Colorado State Univ. Hydrology Paper, 3.

Charlier, R., 1987. Approche unifiée de quelques problèmes non linéaires de mécanique des milieux continus par la méthode des éléments finis. Thèse de doctorat, Faculté des Sciences Appliquées, Université de Liège.

Charlier, R., Radu, J.P., 1997. Hydro-mechanical coupling and strain localisation. Final Proc. NAFEMS World Congress. NAFEMS, Glasgow, pp. 299-310.

Charlier, R., Radu, J.P., Barnichon, J.D., 1997. Water movement effect on strain localisation during a biaxial compression. In: Pietruszczak, S., Pande, G.N. (Eds.), Final Proc. NUMOG Congress Montreal. Balkema, Rotterdam, pp. 219-224.

CIEMAT Report, CEC Contract FI2W-CT91-0102 (DOEO), 1994. Modelling and validation of the thermal-hydraulic-mechanical and geochemical behaviour of the clay barrier, Final Report 1991-1994. CIEMAT. DIRECCIÓN DE TECNOLOGÍA. TÉCNICAS GEOLÓGICAS. Madrid.

Delage, P., 1987. Aspects du comportement des sols non saturés. Rev. Fr. Géotech. 40, 33-43.

Edlefsen, N.E., Anderson, A.B.C., 1943. Thermodynamics of soils moisture. Hilgardia 15 (2), 31-298.

EUR Report, 1996. Thermo-hydraulic-mechanical and geochemical behaviour of the clay barrier in radioactive waste repositories (model development and validation), Final Report. DirectorateGeneral. Science, Research and Development, EUR 16744, EN 1996.

Fredlund, D.G., Morgenstern, N.R., 1977. Stress state variables for unsaturated soils. J. Geotech. Eng. Div. A.S.C.E. 103 GT5, 447-466.

Fredlund, D.G., Rahardjo, H., 1993. Soil Mechanics for Unsaturated Soils. A Wiley-Interscience Publication, John Wiley \& Sons Inc., New York.

Jennings, J.E.B., Burland, J.B., 1962. Limitations to the use of effective stresses in partly saturated soils. Géotechnique 12 (2), 125-144.

Olivella, S., Carrera, J., Gens, A., Alonso, E.E., 1994. Nonisothermal multiphase flow of brine and gas through saline media. Transp. Porous Media 15, 271-293.

Panday, S., Corapcioglu, M.Y., 1989. Reservoir transport equations by compositional approach. Transp. Porous Media 4, 369-393.

Philip, J.R., de Vries, D.A., 1957. Moisture movement in porous materials under temperature gradients. Trans. Am. Geophys. Un. $38,222-232$.

Schrefler, B.A., Simoni, L., Li, X.K., Zienkeiwicz, O.C., 1990. Mechanics of partially saturated porous media. In: Desai, C.S., Gioda, G. (Eds.), Numerical Methods and Constitutive Modelling in Geomechanics. CISM Courses Lect., vol. 311. Springer Verlag, Heidelberg, pp. 169-209.

Thomas, H.R., He, Y., 1995. Analysis of coupled heat, moisture and air transfer in a deformable unsaturated soil. Géotechnique 45 (4), 677-689.

UPC Report, 1996. THM Laboratory tests in FEBEX phase 3. 70UPC-L-3-01. Barcelona. 